\title{
Análise comparativa de pressões horizontais durante o carregamento em silos verticais metálicos
}

\author{
Ariadne Soares Meira ${ }^{1}$ (D), José Wallace Barbosa do Nascimento ${ }^{1,2,3}$ (D), Valneide \\ Rodrigues da Silva ${ }^{3}$ (D), Rafael Torres do Nascimento ${ }^{1}$ (D) \& Hygor Rodrigues \\ Soares $^{1}$ (D)
}

(1) Universidade Federal de Campina Grande, Centro de Tecnologia e Recursos Naturais, Unidade Acadêmica de Engenharia Agrícola, Programa de Pós-Graduação em Engenharia Agrícola, Avenida Aprigio Veloso 882, Bodocongó 58100-000, Campina Grande, Paraíba, Brasil. E-mail: ariadnesm_eng@hotmail.com, cgtorresrafael@gmail.com, hygor2._soares@hotmail.com

(2) Universidade Federal de Campina Grande, Centro de Tecnologia e Recursos Naturais, Unidade de Engenharia Agrícola, Avenida Aprigio Veloso 882, Bodocongó 58100-000, Campina Grande, Paraíba, Brasil. E-mail: wallacebosa@hotmail.com

(3) Universidade Federal de Campina Grande, Centro de Ciências e Tecnologia, Programa de PósGraduação em Engenharia de Processos, Avenida Aprigio Veloso, Bodocongó 58100-000, Campina Grande, Paraíba, Brasil. E-mail: rval707@gmail.com

Meira A.S., Nascimento J.W.B., Silva V.R., Nascimento R.T. \& Soares H.R. (2020) Análise comparativa de pressões horizontais durante o carregamento em silos verticais metálicos. Pesquisa e Ensino em Ciências Exatas e da Natureza, 4: e1539. http://dx.doi.org/10.29215/pecen.v4i0.1539

Editor acadêmico: Angel Ramon Sanchez Delgado. Recebido: 04 setembro 2020. Aceito: 30 outubro 2020. Publicado: 15 dezembro 2020.

Resumo: Os silos são utilizados para armazenamento de grão e/ou produtos pulverulentos alimentícios mantendo as características físicas dos produtos armazenados. Para tanto, o estudo das pressões durante o processo de carregamento, armazenamento e descarregamento do produto são imprescindíveis para manutenção da integridade do silo. $\mathrm{O}$ trabalho tem por objetivo a comparação das pressões horizontais durante o processo de carregamento entre a situação de silo baixo (relação altura/diâmetro $=\mathrm{h} / \mathrm{d}=1$ ) e silo esbelto (relação altura/diâmetro $=\mathrm{h} / \mathrm{d}=4$ ). Para tanto foi utilizado um silo vertical elevado, composto por corpo e tremonha em aço inox com $2.50 \mathrm{~m}$ de altura e $0.64 \mathrm{~m}$ de diâmetro com $10^{-3} \mathrm{~m}$ de espessura. Para aquisição dos dados, foram utilizadas células de carga fixadas a parede do silo e conectadas ao datalogger Spider 8.0, com dados processados pelo Catman 4.5. O produto armazenado foi o milho com repetição do processo de carregamento em quintuplicata para as duas relações h/d. Por resultados obteve-se que quanto maior a relação h/d, maiores as pressões durante o carregamento na área mais próxima a zona de transição entre o corpo do silo e a tremonha. Na relação h/d igual a 1, a representação das pressões horizontais foi praticamente constante.

Palavras chave: Silos verticais, pressões de carregamento, relação h/d.

\section{Comparative analysis of horizontal pressures during loading in metallic vertical silos}

Abstract: The silos are used for the packaging of grain and/or food powders that can be marketed in the periods between harvest, because it maintains the physical characteristics of the stored products. Therefore, the study of the individual flow properties and the characteristics internal to the silo during the process of loading, storing and unloading the product is essential to maintain the integrity of the silo. Thus, the present study aims to compare the horizontal pressures during the loading process between the low silo situation ( $\mathrm{h} / \mathrm{d}=1 \mathrm{ratio})$ and sly silo $(\mathrm{h} / \mathrm{d}=4 \mathrm{ratio})$. For this purpose, a high vertical silo was used, composed of body and hopper in stainless steel with $2.50 \mathrm{~m}$ height and $0.64 \mathrm{~m}$ in diameter with $10^{-3} \mathrm{~m}$ thickness. For data acquisition, load cells fixed to the silo wall were used and connected to the Spider 8.0 datalogger, with data processed by Catman 4.5. The stored product was corn with repetition of the loading process in 
quintuplicate for the two $\mathrm{h} / \mathrm{d}$ ratios. In the $\mathrm{h} / \mathrm{d}$ ratio equal to 1 , the representation of horizontal pressures was practically constant.

Key words: Vertical silos, loading pressures, h/d ratio.

\section{Introdução}

O silo é uma estrutura viável para estocagem dos grãos produzidos e útil para distribuição dos grãos no mercado interno por preservar todas as características físicas e biológicas dos grãos.

Nos diversos estudos que precedem a elaboração de projetos e construção de novos silos, fatores como a magnitude e distribuição das pressões durante a carga e a descarga ainda apresentam algumas incógnitas, uma vez que se trata de um fenômeno complexo em que as pressões dependem, entre outras coisas, da maneira como o produto armazenado flui, da concentricidade ou excentricidade do carregamento e descarregamento deste e das características dos produtos armazenados.

Diversos trabalhos têm por objetivo conhecimento e a determinação dos fenômenos característicos gerados durante o fluxo no carregamento e descarregamento de um silo metálico. O trabalho elaborado por Wojcik et al. (2017) aponta alguns dos fenômenos que podem ser verificados como: o aumento das tensões de parede do silo, diferentes padrões de fluxo, formação e propagação de zonas de cisalhamento no interior do silo e flutuação de pressões, assim como a influência direta da relação altura/diâmetro (h/d) no padrão de fluxo e nas pressões internas.

Com relação as pressões que o produto armazenado exerce sobre a parede do silo, Janssen (1895) considerou a distribuição uniforme desta ao redor de toda a circunferência, sendo tal afirmativa amplamente adotada pelas normas de silo vigentes, sendo possível a simetria nas pressões em torno de todo o perímetro durante o carregamento e descarregamento do silo (Goodey 2017).

Wright (2017) alerta para o fato de que o tipo de carregamento afeta diretamente nas pressões exercidas por produtos granulares sobre as paredes do silo esbelto, havendo alterações nas pressões durante a transição do fluxo mássico podendo haver intermitências para o fluxo misto em algumas ocasiões.

Conforme Coelho (2016), as pressões de carregamento apresentam distribuição simétrica aplicada em todos os pontos verticais do silo que estejam em contato com o produto armazenado, embora haja acréscimo nos valores decorrentes da profundidade. Também chamadas de pressões estáticas, as pressões no carregamento são distribuídas entre pressão horizontal à parede do silo, pressão vertical à parede e pressão de atrito no contato do produto com a parede.

Com relação aos estudos realizados sobre as pressões em silos, pesquisadores como Couto et al. (2012), Ding et al. (2013), Nascimento et al. (2013), Gallego et al. (2015) e Horabik \& Molenda (2017), que utilizam silos protótipos para simulações de ações de carregamento, armazenamento e descarregamento, porém, asseguram que, pelo fato de existirem diversas configurações de silo, materiais de parede, excentricidade de orifício de descarga e produtos a serem armazenados, assim como o pouco conhecimento das ações durante o descarregamento, são necessárias constantes pesquisas experimentais para que as afirmativas sejam fundamentadas.

Assim, o presente trabalho traz por objetivo a comparação entre as pressões horizontais determinadas experimentalmente em um silo vertical com duas configurações de esbeltez distintas (silo baixo e silo esbelto) e as pressões determinadas empiricamente através das normas AS 3774 (1990) e BS EN 1991:4 (2006).

\section{Material e Métodos}


O experimento foi conduzido no Laboratório de Beneficiamento de Sementes (anexo ao Laboratório de Armazenamento e Processamento de Produtos Agrícolas) e Laboratório de Construções Rurais e Ambiência da Universidade Federal de Campina Grande, Campus I Campina Grande/PB utilizando grãos de milho adquiridos na Conab regional, com teor de umidade médio de $12.5 \%$ e peso específico entre 6.9 e $7.9 \mathrm{kN} \cdot \mathrm{m}^{-3}$, apresentando uniformidade granulométrica.

O corpo do silo protótipo foi confeccionado em aço inox com $1 \mathrm{~mm}$ de espessura de parede, apresentando dimensões de $2.50 \mathrm{~m}$ de altura e $0.64 \mathrm{~m}$ de diâmetro e volume de 0.8042 $\mathrm{m}^{3}$ com tremonha, também confeccionada em aço inox com $1 \mathrm{~mm}$ de espessura de parede, tendo inclinação com a horizontal de aproximadamente $60^{\circ}$ e orifício de descarga com diâmetro igual a $0.10 \mathrm{~m}$, concêntrico, com descarregamento por gravidade. $\mathrm{O}$ conjunto corpo e tremonha do silo totalizam um volume armazenável de $0.8636 \mathrm{~m}^{3}$, onde para o milho com um peso específico médio de $7.5 \mathrm{kN} . \mathrm{m}^{-3}$, tem-se aproximadamente $700 \mathrm{~kg}$ de grãos de milho armazenado.

Para as configurações das relações $\mathrm{h} / \mathrm{d}=4 \mathrm{e} \mathrm{h} / \mathrm{d}=1$, o silo foi carregado instantaneamente em duas situações distintas. $\mathrm{Na}$ primeira situação, foi carregado completamente caracterizando o silo com relação h/d igual a 4 e pelas normas AS 3774 (1990) e BS EN 1991:4 (2006) classificado como silo esbelto (Figura 1A). Na segunda situação, o silo foi carregado até alcançar a relação h/d igual a 1 , sendo classificado como silo baixo pelas normas em questão (Figura 1B).

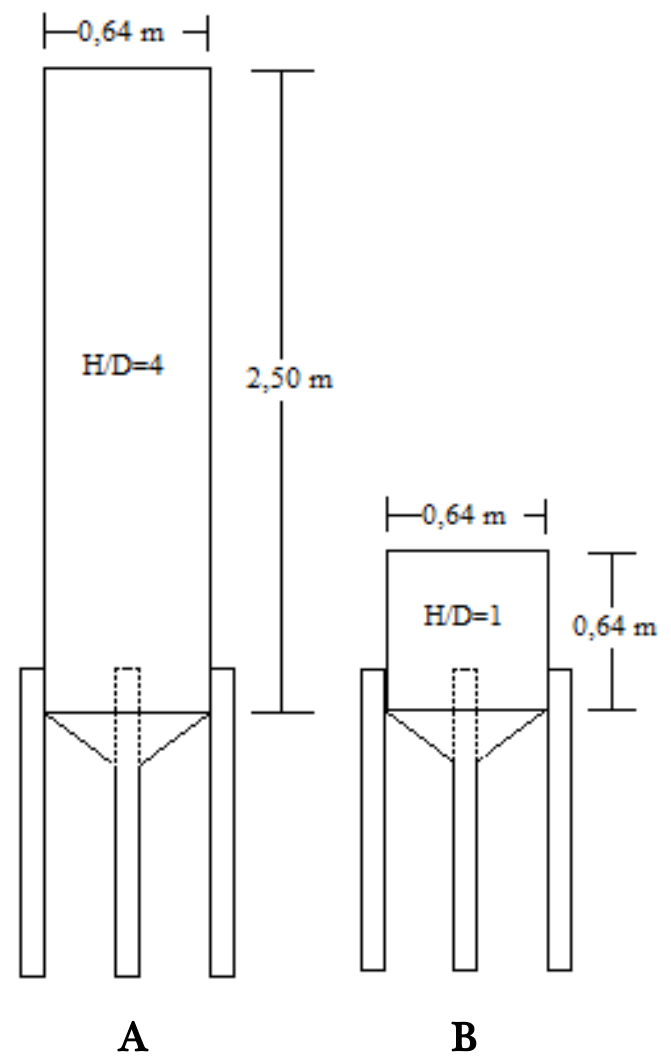

Figura 1. Silo carregado em situações distintas: A. Silo configurado relação h/d igual a 4; B. Silo configurado relação h/d igual a 1 .

No corpo do silo foram realizados sete cortes (Figura 2A) em seções quadradas com dimensões de $0.098 \times 0.098 \mathrm{~m}$ (Figura 2B), todas alinhadas verticalmente para fixação dos sensores de pressão ao longo do corpo do silo.

Nestas seções foram instalados dispositivos compostos por pares de células de carga, espaçados, conforme Figura 2A, de maneira que possam seguir a assíntota de distribuição das pressões, existindo uma relação inversamente proporcional entre a altura do silo e as pressões. 


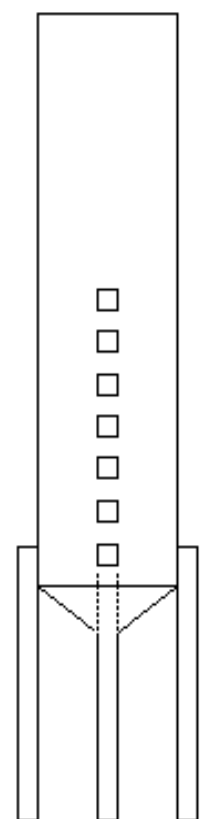

A

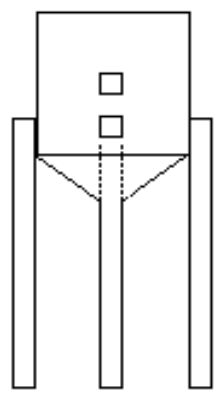

B

Figura 2. Silos com seções extraídas para instrumentação: A. Relação h/d = 4; B. Relação h/d = 1 .

Para a instrumentação do silo foram utilizados dispositivos compostos por pares de células de carga (Figura 3A) modelo TAL223 em liga de alumínio, do fabricante HXASTLP $\mathrm{Hi}$ Sensor, com alimentação entre 5 e $12 \mathrm{Vdc}$, e sinal de saída de $1.0 \mathrm{mV} / \mathrm{V}$ (Figura 3B), conectadas ao sistema de aquisição de dados Spider 8 , do fabricante HBM, configurados para operar em uma frequência de $5 \mathrm{~Hz}$ coletando e armazenando cinco dados por segundo durante toda a execução dos ensaios. O software utilizado para análise dos dados foi o Catman, versão 4.5.

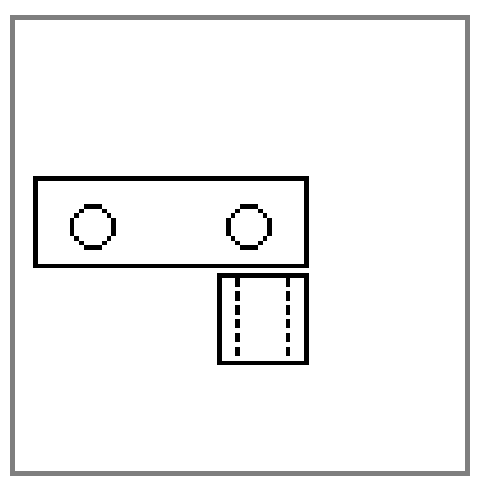

A

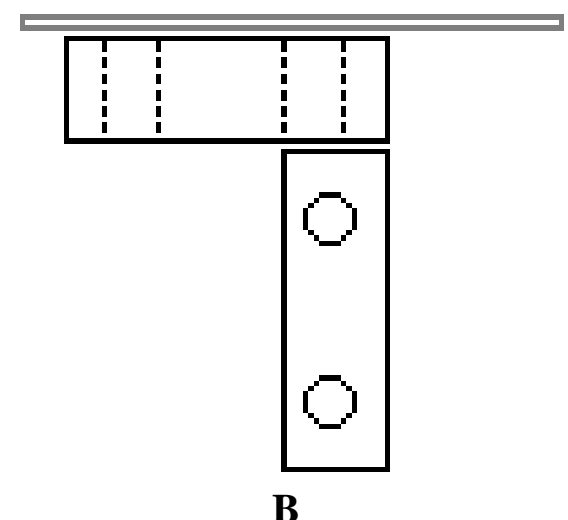

B

Figura 3. Instrumentação do silo com a utilização de dispositivos compostos por pares de células de carga: A. Vista frontal do conjunto de células de carga; B. Vista superior do conjunto de células de carga.

Para a determinação das propriedades de fluxo tais como o ângulo de atrito interno ( $\left.\phi_{i}\right)$, efetivo ângulo de atrito $\left(\phi_{e}\right)$ e o ângulo de atrito do produto com a parede $\left(\phi_{w}\right)$ utilizou-se o aparelho de cisalhamento direto por translação modelo TSG 70-140, conhecido como "Jenike Shear Cell'. Este aparelho utilizado para os ensaios é composto por uma célula de cisalhamento de forma cilíndrica, dividida em um anel inferior e um superior, que são preenchidos com a amostra do produto a ser cisalhada, um pendural com pesos para a aplicação da força vertical na amostra e uma haste para aplicação da força cisalhante.

Para a determinação das pressões horizontais foram utilizadas equações contidas nas normas estrangeiras AS 3774 (1990) e BS EN 1991:4 (2006). 
Na norma BS EN 1991:4 (2006) a pressão horizontal está diretamente relacionada a raio hidráulico do silo e ao coeficiente de atrito do material com o qual ele é confeccionado (Eq. 1):

$$
\operatorname{Phf}(z)=\operatorname{PhoYj}(z) \quad(\text { Eq. } 1)
$$

Onde: $P h f(z)$ é a pressão horizontal durante o carregamento; Pho é a pressão horizontal inicial (kPa) (Eq. 2) que leva em consideração o peso específico consolidado do produto armazenado $\gamma\left(\mathrm{kN} / \mathrm{m}^{3}\right)$, o fator $k$ que é a relação teórica entre as pressões horizontais e verticais que atuam no silo, além do zo $(\mathrm{m})$ que é a profundidade característica da massa de grãos (Eq. 3)

$$
\begin{gathered}
\text { Pho(z) }=\gamma K z o \\
Z o=\frac{1}{K \mu} \frac{A}{U}
\end{gathered}
$$

O parâmetro zo é determinado relacionando-se o raio hidráulico $\frac{A}{U}$ com A a área do silo e U o perímetro do silo, e o inverso do fator $k$ pelo coeficiente de atrito entre o produto e o material da parede do silo $\mu$.

A pressão horizontal segundo esta norma ainda utiliza o coeficiente de variação de profundidade $Y j(z)$ que leva em consideração a razão entre a profundidade efetiva e a profundidade característica da massa de grãos armazenados (Eq. 4).

$$
\left.Y j(z)=1-e^{\left(-\frac{z}{z o}\right)} \quad \text { (Eq. } 4\right)
$$

Já a norma AS 3774 (1990) leva em consideração para a equação que determina as pressões horizontais o peso específico consolidado e o coeficiente de dimensão do silo (Eq. 5), assim como o coeficiente de sobrepressão $\mathrm{Cz}$ característico (Eq. 6):

$$
\begin{aligned}
P h f & =\gamma r c \mathrm{Cz} / \mu \\
C z & =1-e^{\left(-\frac{z}{z o}\right)}
\end{aligned}
$$

Onde: $P h f$ é a pressão horizontal durante o carregamento $(\mathrm{kPa}) ; \gamma$ o peso específico consolidado $\left(\mathrm{kN} / \mathrm{m}^{3}\right) ; r c$ um coeficiente de dimensão característica do silo, que neste caso é igual a $0.25 \mathrm{dc}$ (dc = diâmetro efetivo do silo); $\mathrm{Cz}$ como sendo o coeficiente de profundidade de Janssen, que leva em consideração a razão entre a profundidade efetiva e a profundidade característica da massa de grãos; e $\mu$ como o coeficiente de atrito entre o produto e o material da parede do silo.

\section{Resultados e Discussão}

Para a determinação empírica das pressões horizontais, segundo as normas AS 3774 (1990) e BS EN 1991:4 (2006), foram realizados testes para determinação das propriedades físicas e de fluxo do produto em estudo apresentados na Tabela 1 com limites inferior (Inf) e superior (Sup).

Tabela 1. Propriedades de fluxo de grãos de milho. $\boldsymbol{\gamma}=$ peso específico consolidado $\left(\mathrm{kN} \mathrm{m}_{-}{ }^{3}\right) ; \boldsymbol{\phi}_{\boldsymbol{i}}=$ ângulo de atrito interno $\left({ }^{\circ}\right) ; \phi_{e}=$ efetivo ângulo de atrito $\left(^{\circ}\right) ; \phi_{w}=$ ângulo de atrito do produto com a parede $\left(^{\circ}\right) ; C=$ coesão $(\mathrm{Pa})$.

\begin{tabular}{ccccccccc}
\hline \multicolumn{2}{c}{$\gamma$} & \multicolumn{2}{c}{$\phi_{i}$} & \multicolumn{2}{c}{$\phi_{e}$} & $\phi_{w}$ & \multicolumn{1}{c}{$\boldsymbol{C}$} \\
Inf & Sup & Inf & & Sup & Inf & Sup & & \\
\hline 6.5 & 7.2 & 11.8 & 20.0 & 18.0 & 22.2 & 8.0 & 362.4 \\
\hline
\end{tabular}


Com relação as pressões, levando em consideração as Figuras 4 e 5 que representam as assintotas das pressões horizontais instantâneas, durante o carregamento do silo com as duas configurações h/d igual a 4 (Figura 4) e h/d igual a 1 (Figura 5), podemos afirmar que, as pressões experimentais são inferiores as pressões normatizadas, pois estas possuem um coeficiente de segurança adicionado ao coeficiente de sobrepressão que pode aumentar consideravelmente os limites máximos encontrados durante o experimento.

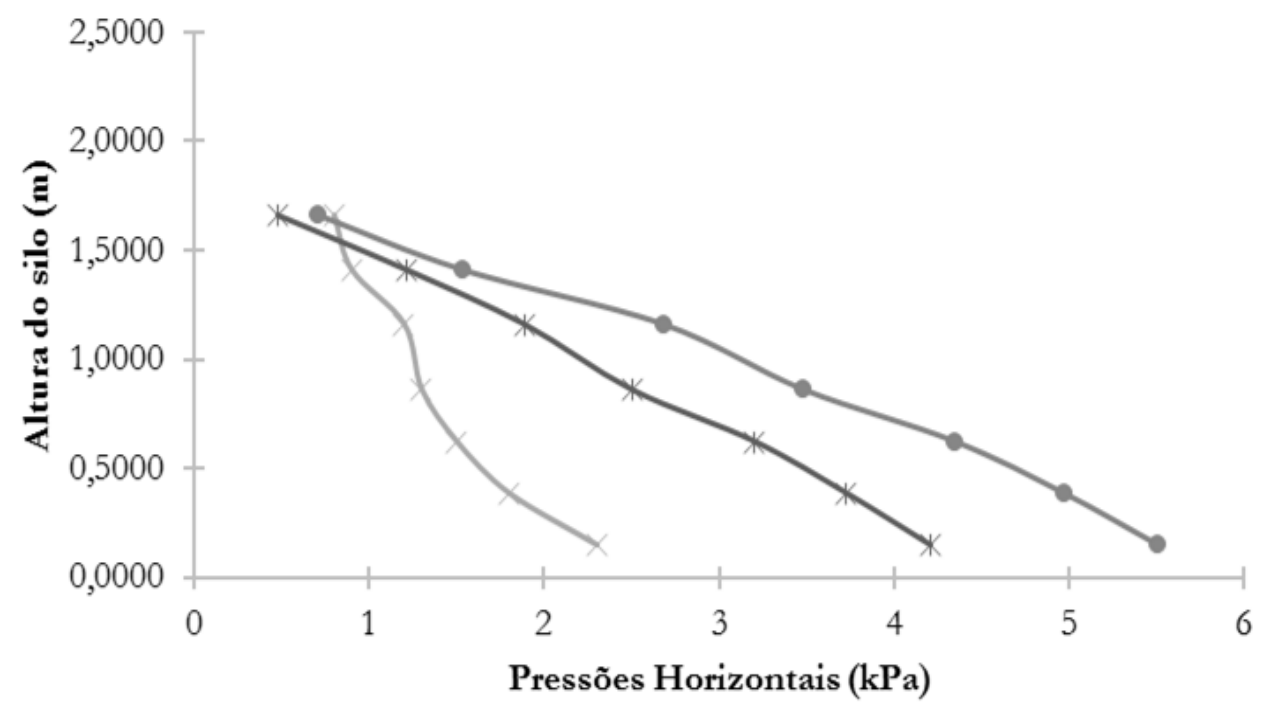

$\longrightarrow$ EXPERIMENTAL - $\mathrm{C} \rightarrow$ AUSTRALIANA - C $\multimap$ EUROPEIA - C

Figura 4. Pressão horizontal com $\mathrm{h} / \mathrm{d}=4$ (experimental versus normatizada).

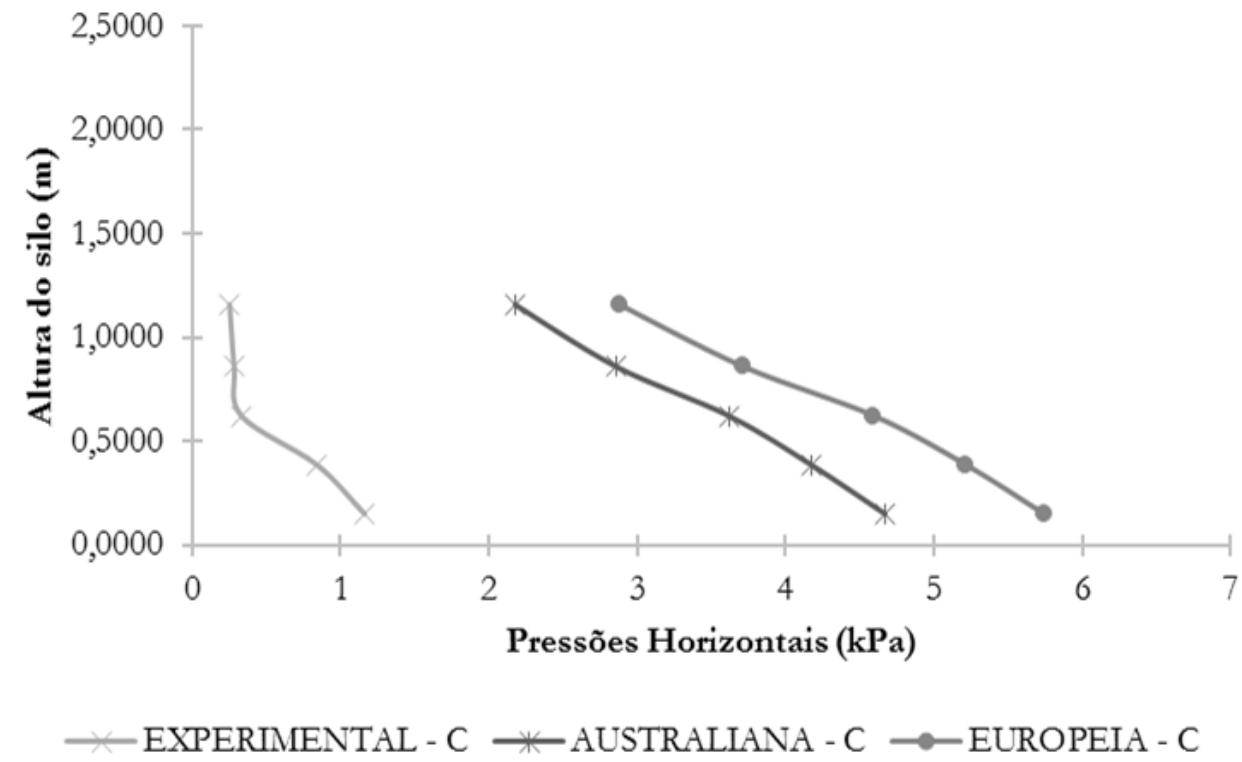

Figura 5. Pressão horizontal com $\mathrm{h} / \mathrm{d}=1$ (experimental versus normatizada).

Ainda ao analisar a Figura 4, percebe-se que as pressões na zona livre são semelhantes tanto no estudo experimental quanto nos estudos empíricos normatizados, porém, ao longo do processo de carregamento, as pressões experimentais tendem a não serem tão linear quanto a representação das pressões horizontais normatizadas.

Couto et al. (2013) ao instrumentarem um silo protótipo com células de carga e compararem as assintotas experimentais com as normatizadas na BS EN 1991:4 (2006), encontram valores experimentais inferiores aos normatizados, sendo aberto um parêntese ao 
fato de que foram utilizados dois processos distintos para carregamento e descarregamento do silo e, em ambos, os valores normatizados se sobressaíram.

Na Figura 5, o silo com relação $\mathrm{h} / \mathrm{d}=1$ apresenta valores de até $80 \%$ de inferioridade, quando comparados as normas em estudo, quando a zona em questão é imediatamente superior a zona de transição entre o corpo do silo e a tremonha.

Wang et al. (2015) por sua vez, ao realizarem um trabalho com simulação de pressões, encontram valores de pressões experimentais próximos aos valores obtidos através da simulação e até $20 \%$ inferiores aos valores a partir da norma BS EN 1991:4, sendo possível assim afirmar que, para a situação imposta, a simulação representa perfeitamente as ações causadas pelo produto armazenado as paredes do silo.

Quando realizada a comparação dos valores experimentais entre si, levando em conta a relação $\mathrm{h} / \mathrm{d}$, é de fácil percepção que quanto maior a relação $\mathrm{h} / \mathrm{d}$, maiores as pressões horizontais no interior do silo, principalmente na zona que se aproxima do zero (ponto considerado na zona de transição entre o corpo do silo e a tremonha) pois há uma quantidade maior de produto sobre esta camada (Tabela 2).

Tabela 2. Pressão x Altura da zona no silo.

\begin{tabular}{lcc}
\hline & $\mathrm{H} / \mathrm{D}=\mathbf{4}$ & $\mathrm{H} / \mathrm{D}=\mathbf{1}$ \\
\cline { 2 - 3 } C7 & 0.40 & - \\
C6 & 0.36 & - \\
C5 & 0.47 & - \\
C4 & 0.60 & - \\
C2 & 0.77 & 0.35 \\
C1 & 0.79 & 0.51 \\
\hline
\end{tabular}

Pelo fato do silo ter sido instrumentado com células de carga em 7 ponto quando a relaçao $\mathrm{h} / \mathrm{d}$ foi igual a 4 e quando a relação $\mathrm{h} / \mathrm{d}$ foi igual a 1, verificamos na Tabela 2 que, na zona livre da massa de grãos, ou seja, na zona surerior, onde não existe outra camada de grãos exercendo pressão vertical sobre esta zona, as pressões horizontais são as menores encontradas durante o processo de carregamento, assim como, quanto mais inferior é a zona de estudo, maiores são as pressões horizontais pois as camadas de grãos durante o carregamento se sobrepõem a esta.

Os valores obtidos experimentalmente ao serem comparados com os valores obtidos através da resolução empírica contida nas normas estrangeiras, observa-se que, a norma européia (BS EN 1991:4 2006) majora os valores de pressões horizontais, sendo os valores normatizados na AS 3774 os que podem se aproximar e representar mais a situação imposta no presente trabalho.

Em antagonismo ao encontrado como resultado nesta pesquisa, Kachurenko \& Bannikov (2016) encontram em seu trabalho, valores aproximados entre pressões horizontais experimentais, normatizadas e geradas por modelos matemáticos utilizando elementos finitos (FEM), afirmando que pode haver representatividade dos valores reais, se a tomada de decisão for semelhante a situação utilizada experimentalmente.

Couto et al. (2012) alertam para o fato de que, ao serem realizados experimentos com silos protótipos, existe uma infinidade de configurações que podem ser adotadas tanto na relação H/D quanto na conformação das paredes do silo, excentricidades de carga e descarga, para que assim, haja a maior precisão possível nos processos de carregamento e descarregamento e maior e melhor utilização dos equipamentos utilizados nestes processos.

\section{Conclusões}

Conclui-se que, as pressões horizontais sofrem influência direta da massa de produto inserida do silo e que quando mais próxima a zona infinitesimal em estudo se aproxima da zona 
de transição entre o corpo do silo e a tremonha, maiores são as pressões horizontais encontradas pois há, neste ponto, a mudança de direção das forças verticais atuantes durante o carregamento, fazendo com que haja um pico de pressão exatamente no início do processo de carregamento, onde há movimento do produto em direção ao orifício de descarga.

Conclui-se ainda que a norma estrangeira australiana (AS 3774) e européia (BS EN 1991:4), embora sejam largamente utilizadas para elaboração de projeto de silos, possam não representar fidedignamente o comportamento dos grãos no interior do silo, por existirem situações particulares onde os grãos, o meio externo e a configuração do silo podem apresentar variações durante o processo de carregamento, armazenamento e descarregamento dos produtos.

\section{Agradecimentos}

Ao Laboratório de Beneficiamento de Sementes e Laboratório de Construções Rurais e Ambiência pela concessão do espaço e equipamentos para execução do trabalho e a Coordenação de Aperfeiçoamento de Pessoal de Nível Superior (Capes) pela concessão da bolsa de estudos aos membros envolvidos no trabalho.

\section{Referências}

AS 3774 (1990) Loads on bulk containers. Sydney: Australian Standard. 62 p.

BS EN 1991-4_Eurocode 1 (2006) Actions on structures. Part 4: Silos and tanks. Brussels: The European Union Per Regulation 305/2011, Directive 98/34/EC, Directive 2004/18/EC. 112 p.

Coelho L.C. (2016) Software para cálculo de fluxo e pressões em silos cilíndricos metálicos para armazenamento de produtos agrícolas e industriais. Dissertação (Programa de Pós-Graduação em Engenharia Civil). Escola de Engenharia de São Carlos, São Carlos.

Couto A., Ruiz A. \& Aguado P.J. (2012) Design and instrumentation of a mid-size test station for measuring static and dynamic pressures in silos under different conditions - Part I: Description. Computers and Electronics in Agriculture, 85: 164-173. https://doi.org/10.1016/j.compag.2012.04.009

Couto A., Ruiz A., Herráez L., Moran J. \& Aguado P.J. (2013) Measuring pressures in a slender cylindrical silo for storing maize. Filling, static state and discharge with different material flow rates and comparison with Eurocode 1 part 4. Computers and Electronics in Agriculture, 96: 40-56. https://doi.org/10.1016/j.compag.2013.04.011

Ding S., Rotter J.M., Ooi J.Y., Enstad G. \& Xub D. (2013) Normal pressures and frictional traction son shallow conical hopper walls after concentric filling: Predictions and experiments. Chemical Engineering Science, 89: 264-272.

Gallego E., Ruiz A. \& Aguado P.J. (2015) Simulation of silo filling and discharge using ANSYS and comparison with experimental data. Computers and Electronics in Agriculture, 118: 281289. https://doi.org/10.1016/j.compag.2015.09.014

Goodey R.J., Brown C.J. \& Rotter J.M. (2017) Rectangular steel silos: Finite element predictions of filling wall pressures. Engineering Structures, 132: 61-69. https://doi.org/10.1016/j.engstruct.2016.11.023

Horabik J. \& Molenda M. (2017) Distribution of static pressure of seeds in a shallow model silo. International Agrophysics, 31(2): 167-174. https://doi.org/10.1515/intag-2016-0038

Janssen H.A. (1895) Versuche über Getreidedruck in Silozellen. Volume 39. Düsseldorf: Verein Deutscher Ingenieure. $1049 \mathrm{p}$.

Kachurenko V.V. \& Bannikov D.O. (2016) Experimental study of horizontal pressure distribution on corrugated steel silo walls. Наука та прогрес транспорту. Вісник Дніпропетровського національного університету залізничного транспорту, 64(4): 151-160.

Nascimento J.W.B., Lopes Neto J.P. \& Montross M.D. (2013) Horizontal pressures in cylindrical metal silos and comparison with different international standards. Engenharia Agrícola, 33(4): 601-611. https://doi.org/10.1590/S0100-69162013000400002 
Wang Y., Luc Y. \& Ooi J.Y. (2015) A numerical study of wall pressure and granular flow in a flatbottomed silo. Powder Technology, 282: 43-54. https://doi.org/10.1016/j.powtec.2015.01.078

Wojcik M., Sondej M., Rejowski K. \& Tejchman J. (2017) Full-scale experiments on wheat flow in steel silo composed of corrugated walls and columns. Powder Technology, 311: 537-555. https://doi.org/10.1016/j.powtec.2017.01.066

Wright C. (2017) The Effect of Grain Shape on Side-Wall Pressure in Model Grain Silos. Proceedings of The National Conference: On Undergraduate Research (NCUR). University of Memphis, Memphis. 